\title{
Research Paper \\ Comparison of Impulsivity and Experiential Avoidance in Obese Individuals With and Without Binge Eating Disorder
}

\author{
Leila Kehtari Harzangh ${ }^{1}$, Rasoul Heshmati², *Hamid Poursharifi ${ }^{3}$
}

1. MA., Department of Psychology, Faculty of Education and Psychology, University of Tabriz, Tabriz, Iran.

2. PhD. in Health Psychology, Assistant Professor, Department of Psychology, Faculty of Education and Psychology, University of Tabriz, Tabriz, Iran.

3. PhD. in Health Psychology, Associate Professor, Department of Clinical Psychology, University of Social Welfare and Rehabilitation Sciences, Tehran, Iran.

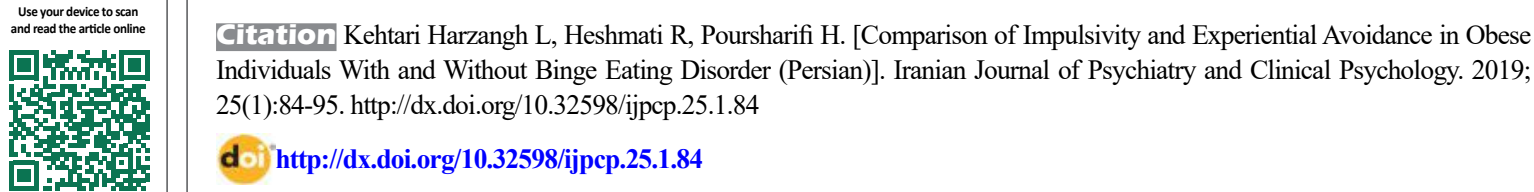

Keywords:

Binge Eating Disorder

Obese, Experiential

avoidance, Impulsivity

\section{A B STRACT}

Objectives This research aimed to compare impulsivity and experiential avoidance in obese individuals with and without Binge Eating Disorder (BED).

Methods We obtained data from 60 severely obese women referring to health centers and gyms. They were selected by convenience sampling method. The participants completed Gormally Binge Eating Scale (BES), Barratt Impulsiveness Scale (BIS), and Bond Acceptance and Action Questionnaire (AAQ). The obtained data were analyzed by the discriminant analysis method.

Results Among 5 investigated components in two groups of with and without binge eating disorder, the components of non-planning impulsivity $(P<0.01)$, and emotional avoidance and control $(P<0.05)$ were found significant.

Conclusion In the two groups of with and without binge eating disorder, the components of non-planning impulsivity, emotional avoidance and control were found significant. This means that significant differences exist between individuals with and without binge eating disorder.

\section{Extended Abstract}

\section{Introduction}

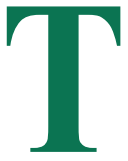

his study aimed to differentiate obese and non-obese people based on impulsivity and avoidance. Without identifying and understanding the psychological factors associated with overeating and obesity, we cannot be successful in preventing obesity. Consequently, studying the mental structures associated with problems in controlling eating behavior can be considered as a useful step towards better understanding and treatment of this disorder.

\section{Method}

The present study design is descriptive correlational based on the purpose of the data collection. The statistical population of this study comprised all women with overweight referring to health houses, sports clubs, and weight loss centers in Tabriz City, Iran.

The inclusion criteria were the willingness to participate in the study, with BMI greater than $30 \mathrm{~kg} / \mathrm{m}^{2}$, and the required literacy to complete the questionnaires. The exclusion criteria included having diagnostic criteria for other eating disorders or other psychiatric disorders, under medications or

* Corresponding Author:

Hamid Poursharifi, PhD.

Address: Department of Clinical Psychology, University of Social Welfare and Rehabilitation Sciences, Tehran, Iran.

Tel: +98 (912) 5264947

E-mail: ha.poursharifi@uswr.ac.ir 
physical conditions affecting their weight. Then, a sample of 210 people was screened based on Binge Eating Scale (BES) for obesity disorder. By calculating the means of the $\mathrm{z}$ index of these 210 people, the mean of those who had a standard deviation above and below and they were selected and entered the second sampling stage. The study used a convenience sampling method.

In the second stage, 33 subjects were selected from obese people who were diagnosed with overeating and were interested in participating in the study. Also, 35 subjects were selected from obesity who received criteria for overdose. In order to determine the minimum sample size in the correlational studies, we used the Fidell and Tabachnick formula as $\mathrm{N} \geq 50+8 \mathrm{M}$. In this study, the second step was based on the number of independent variables (two independent variables) using this formula. For collecting data, Gormally Binge Eating Scale (BES), Barratt Impulsiveness Scale (BIS), and Bond Acceptance and Action Questionnaire (AAQ) were used. For analysis of data in the descriptive statistics section, the central and dispersion indices were used. Regarding the inferential statistics, the audit analysis method was used. All analyses were performed in SPSS version 23.

\section{Results}

The results showed that among the five components of the study, two groups of overweight and overweight disorders were a non-programmatic impulsive component at the level of 0.01 and emotional avoidance and control at the level of 0.05 . Considering the small amount of Welsh lambda and the high amount of the Chi-square and significance level, the audit function has obtained a good diagnostic power for explaining the dependent variables (in two groups). In both groups, obese and non-obese, overweight, is an impulsive, impulsive, emotional avoidance and meaningful control component. This means that there is a significant difference in obesity among obese people who are overweight with those who are overweight and have a significant difference in these components.

\section{Discussion}

On the basis of impulsivity and avoidance variables, two groups of obese and non-obese people with binge eating disorder can be distinguished from each other. In this regard, high levels of immoral behavior can be related to their unsuccessful attempts to reduce overeating and their dietary intake. Also, the impulsivity can be associated with trying unsatisfactory diets. Additionally, overeating behaviors by reducing the effect of negative emotions (such as anger and anxiety) can lead to a brief relief of negative emotional excitement, which in turn can increase the overeating behavior.

\section{Ethical Considerations}

\section{Compliance with ethical guidelines}

All ethical principles were considered in this article. The participants were informed about the purpose of the research and its implementation stages; they were also assured about the confidentiality of their information; Moreover, They were allowed to leave the study whenever they wish, and if desired, the results of the research would be available to them.

\section{Funding}

The present paper was extracted from the MSc. thesis of the first author, in Department of Psychology, Faculty of Education and Psychology, University of Tabriz.

\section{Authors contributions}

All authors contributed in preparing this article.

\section{Conflicts of interest}

The authors declared no conflict of interest. 
This Page Intentionally Left Blank 


\title{
تمايزكذارى افراد هاق مبتلاو غيرمبتلابه اختلال يرخورى بر اساس تكانشكَرى و اجتناب تجربهاى:

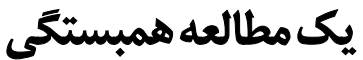

\author{
ليلا كهترى هرزنق '؛ رسول حشمتى! • حميد يورشريفى' \\ 1- كارشناسى ارشد، تروه روانشناسى، دانشكده علوم تربيتى و روانشناسي، دانشعاه تبريز، تبريز، ايران.

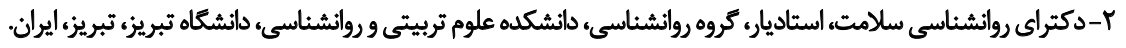

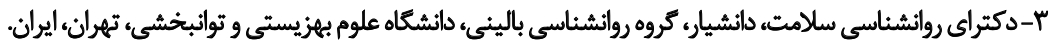

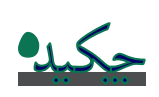

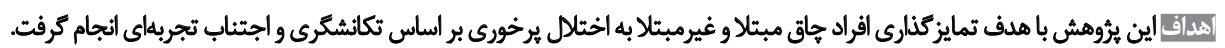

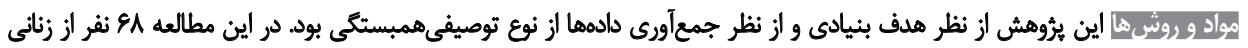

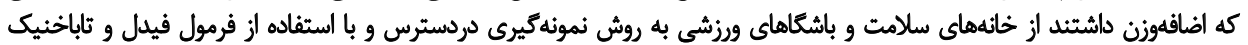

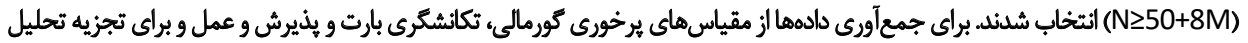

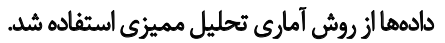

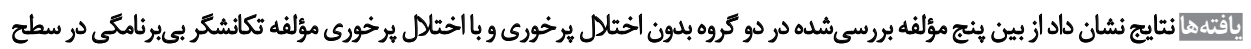

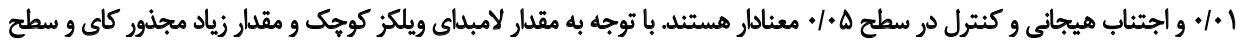

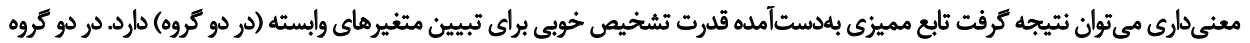

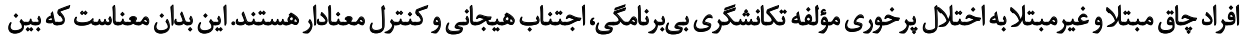

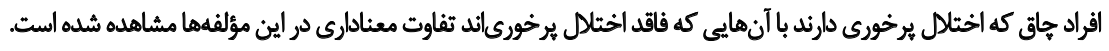

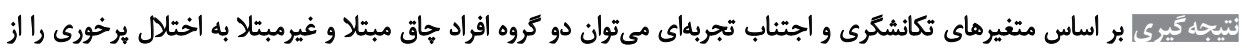
هم تفكيك كرد.
\end{abstract}

تاريخ دريافت: 9 مرداد و

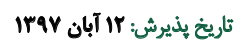

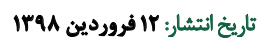

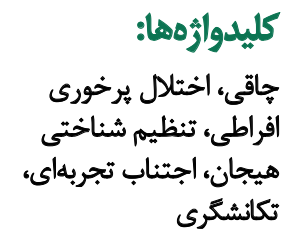

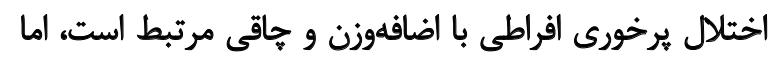

datê

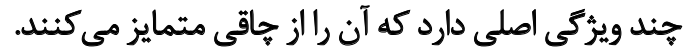

افراد جاق مبتلا به اختلال برخورى افراطى در مقايسه با افراد

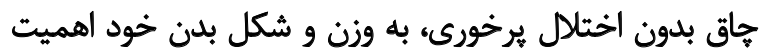

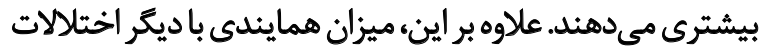

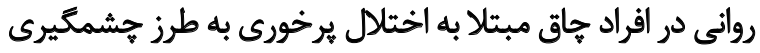

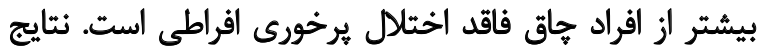

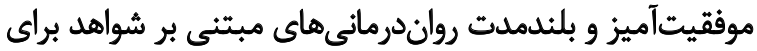

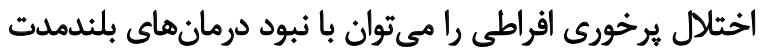

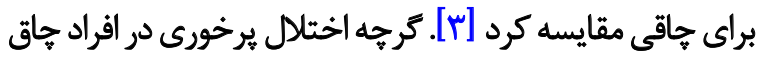

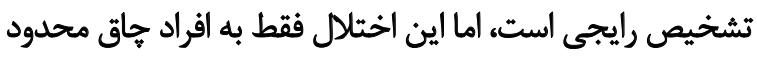

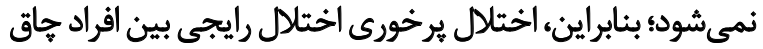
و با اضافهوزن و مشكل اساسى در حيطه سلامت است.

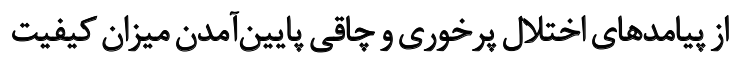

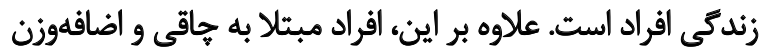

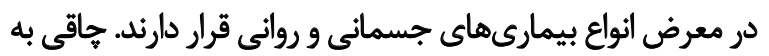

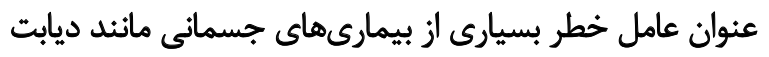

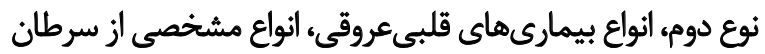

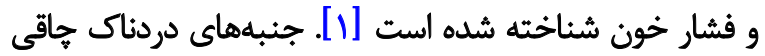

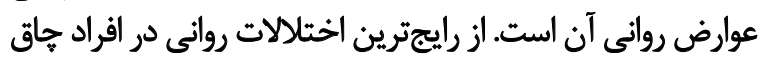

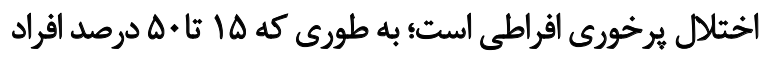

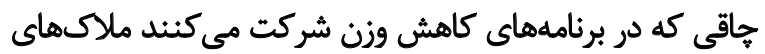

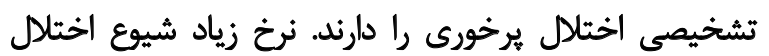

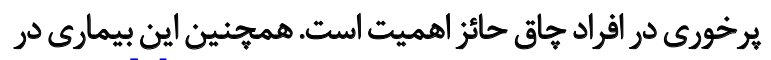

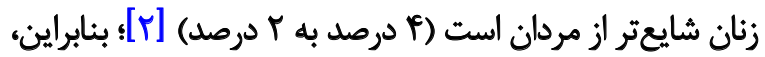

ㅂ.

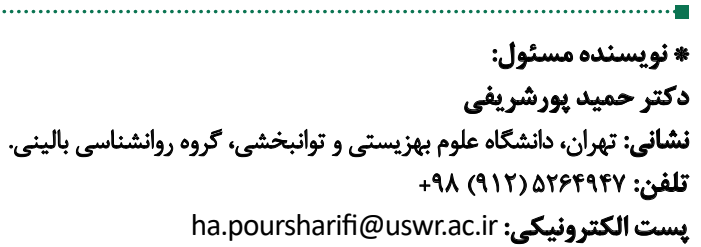




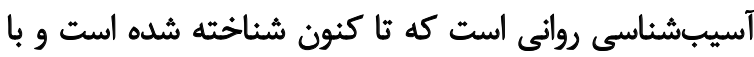

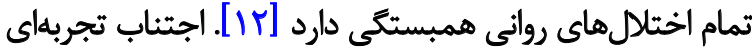

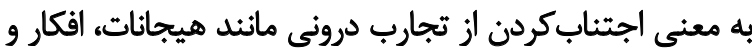

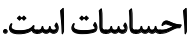

اجتناب تجربهاى اجتناب از محركها عينى يا خارجى نيست،

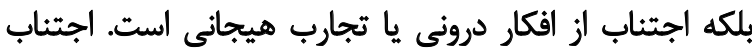

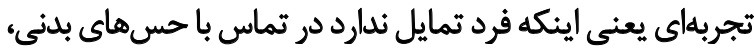

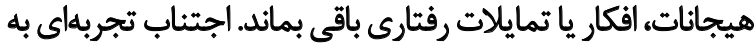

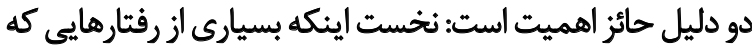

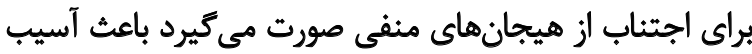

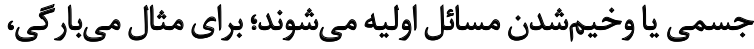

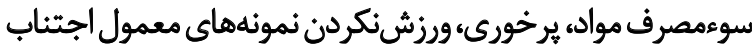

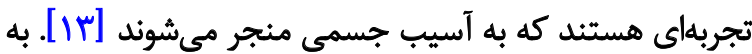

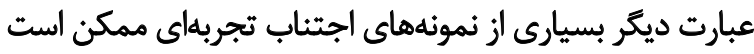

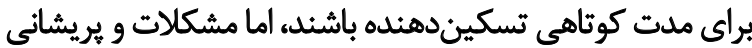

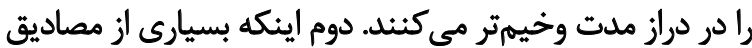

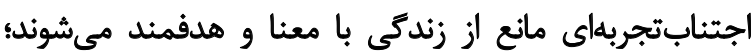

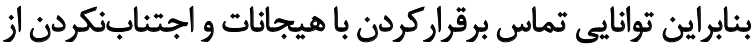

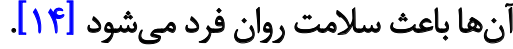

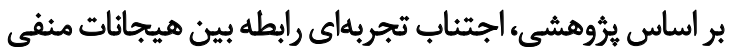

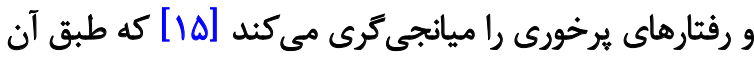

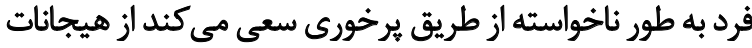

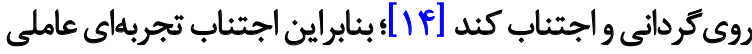

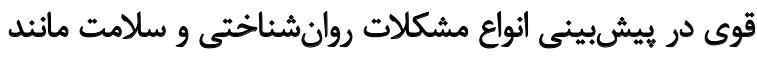

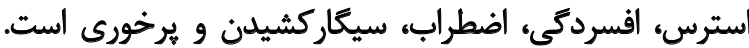

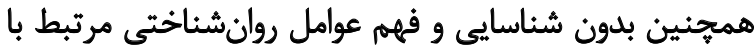

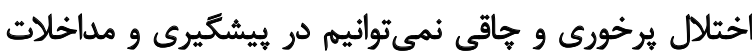

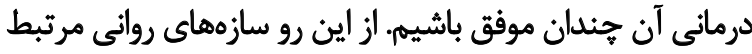

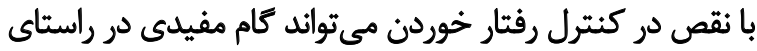

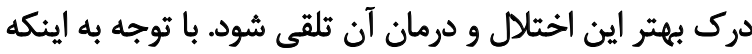

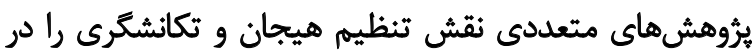

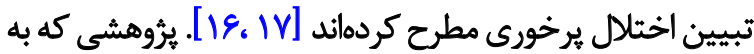

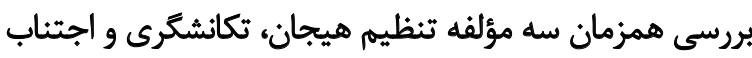
تجربهاي يرداخته باشده يافت نشد.

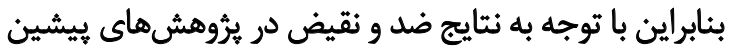

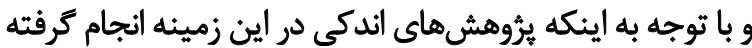

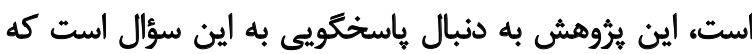

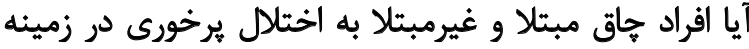
تكانشكرى و اجتناب تجربهاى با هم تفاوت دارند؟

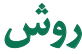

اين يروهش به لحاظ هدف از نوع بنيادى و به لحاظ نحوه

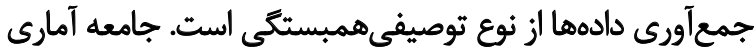

افراد هاق را الز منظرى مي توان به دو دسته تقسيم كرد: افراد

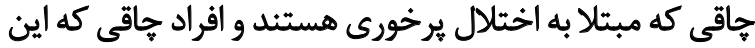

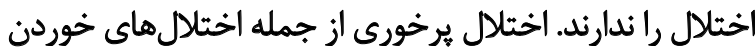

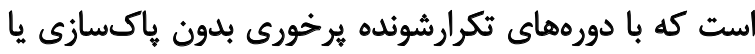

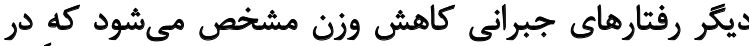

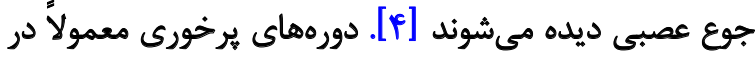

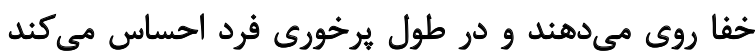

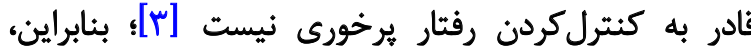

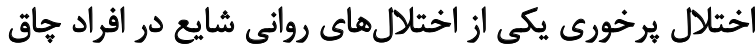

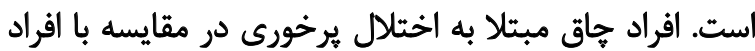

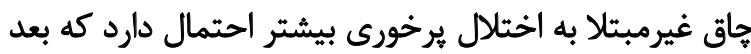

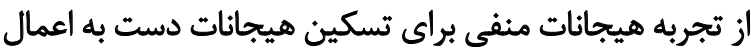

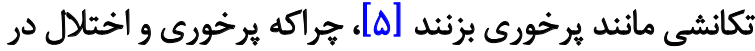

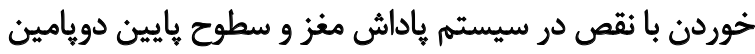

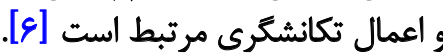

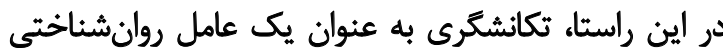

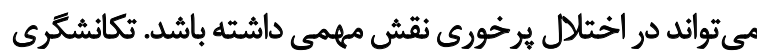

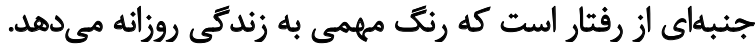

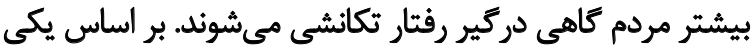

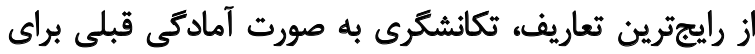

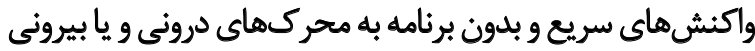

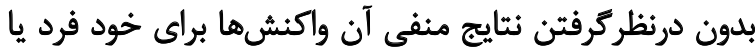

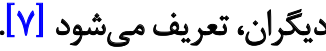

يكى از رايج ترين ديدكاهها در زمينه تكانشكرى كه به آن توجه شده است، نظريه بارت و همكاران است. اين محقدق بات بات تركيب

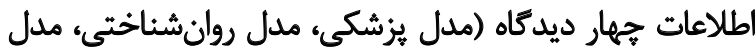

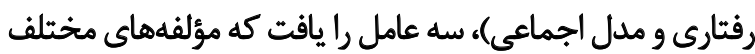

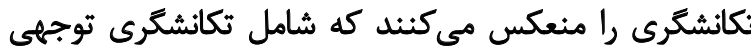

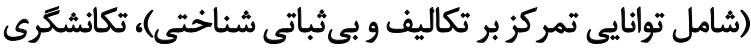

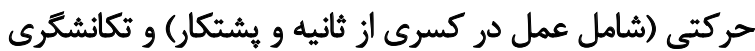

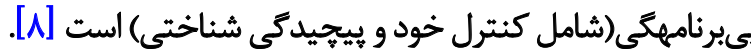

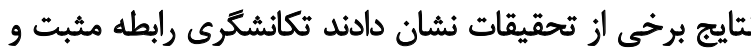

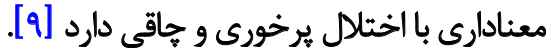
با وجود اين، نتايج مطالعات مختلف در زمينه ارتباط

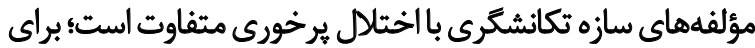

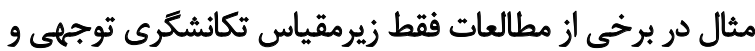

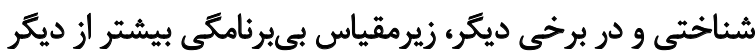

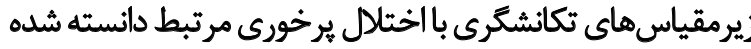

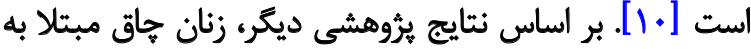

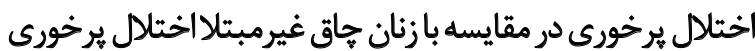

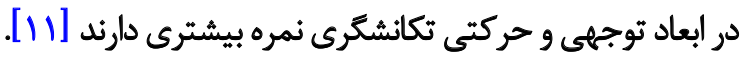
از سوى ديكر اجتناب تجربهاى يكى از فرايندهاى متداول 


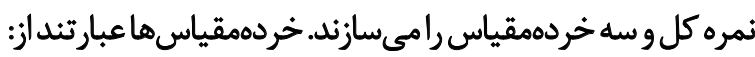

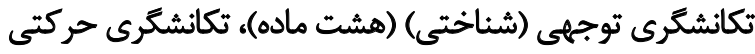

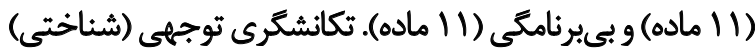

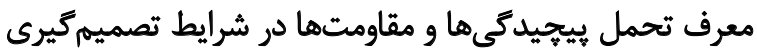

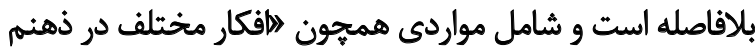

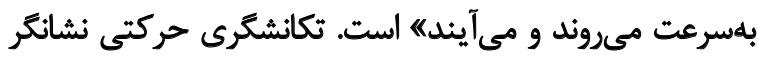

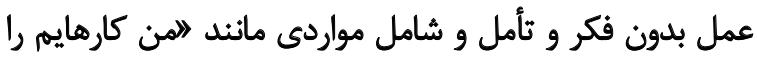
بلدون فكر انجام مي دهمي في است.

تكانشكَرى مبتنى بر بحبرنامكى مبين آيندهنكرنبودن در رفتار

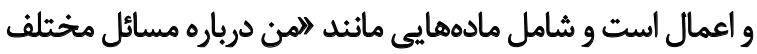

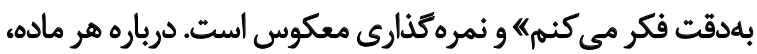

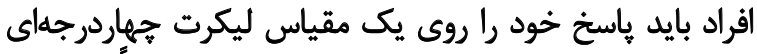

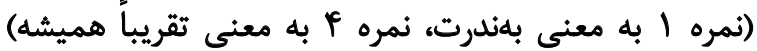

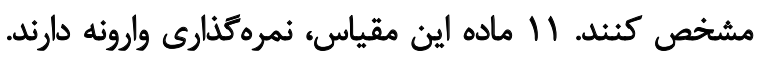

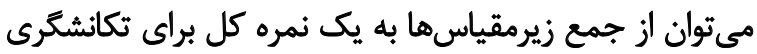

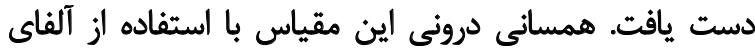

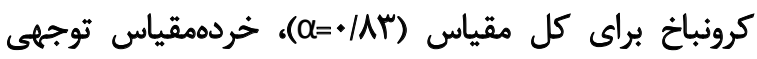
(Q)

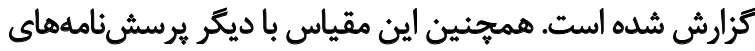

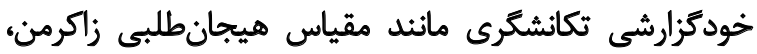

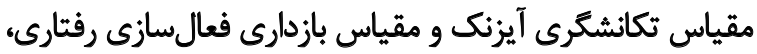

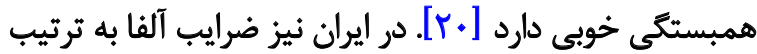

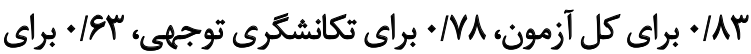

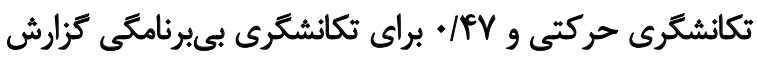

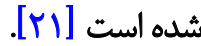

\section{هرسشنامه بذيرش و عمل نسخه دومه}

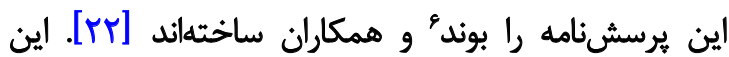

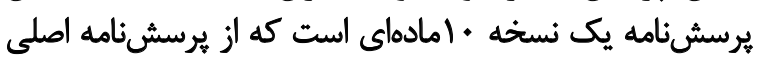

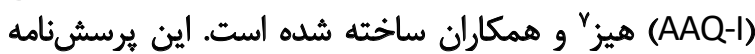

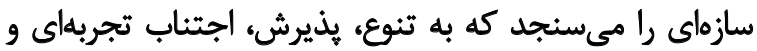

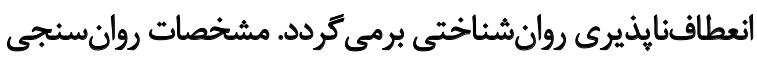

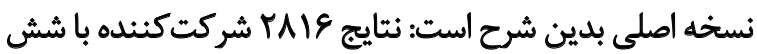

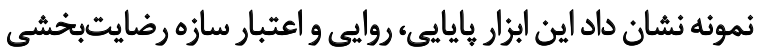

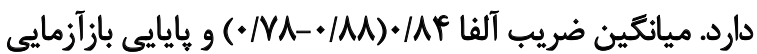

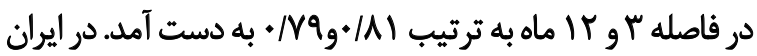

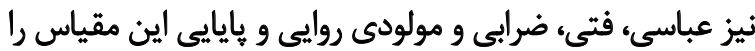

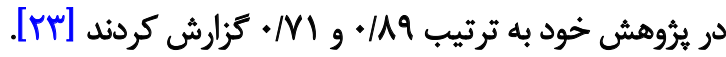
به نظر مىرسد ח-CAQ مفهوم مشابه با I-AAQ را اندازه

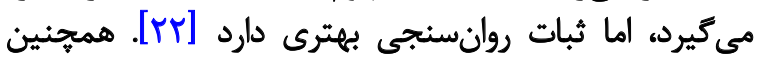

5. Acceptance and Action Questionnaire-II (AAQ-II)

6. Bond

7. Hayes
اين يرُوهش تمام زنانى بودند كه اضافهوزن داشتند و در فاصله

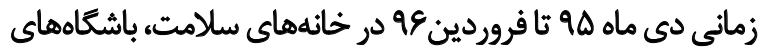

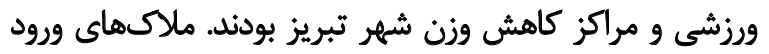

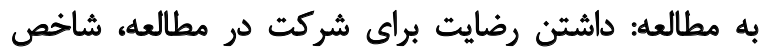

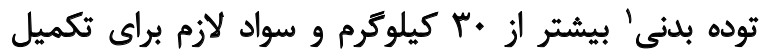

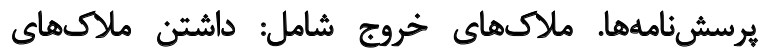

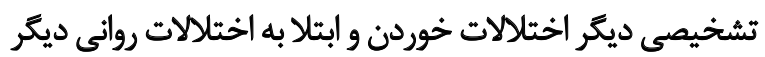

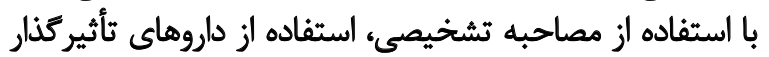
روى وزن و داشتن بيمارىهاى جسمانى تأثيركذار روى وزيه وزن.

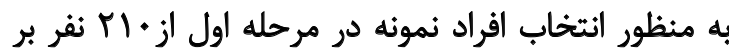

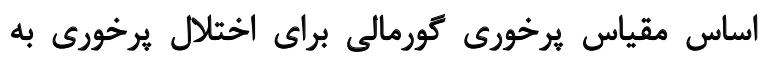

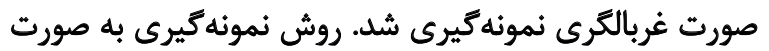

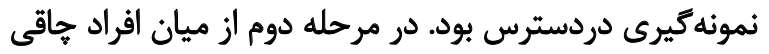

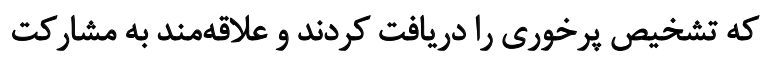

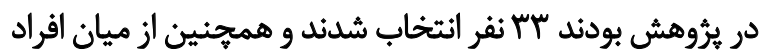

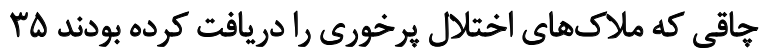

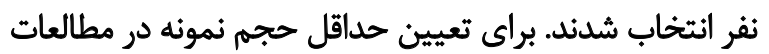

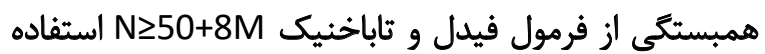

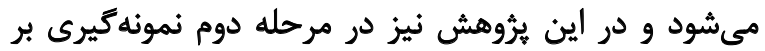

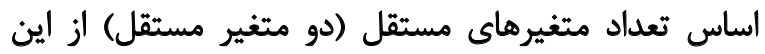

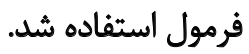

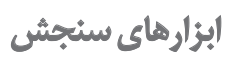

در اين يُروهش ابزارهاى استفادهشده خودكزارشكر بودهاند.

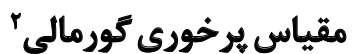

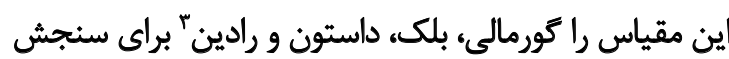

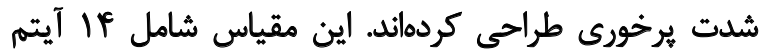

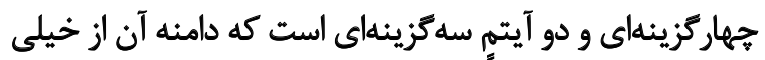

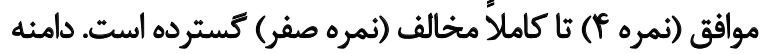

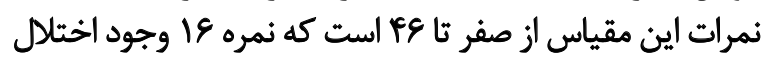

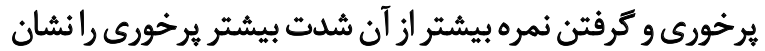

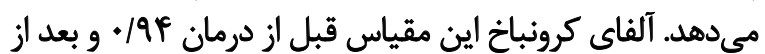

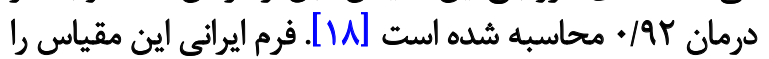

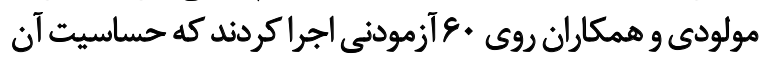

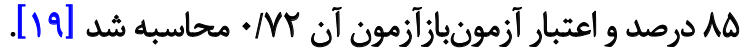

$$
\text { مقياس تكانشَّرى بارت }
$$

مقياس تكانشكُرى بارت از • ماده تشكيل شده است كه يك

\footnotetext{
1. Body Mass Index (BMI)

2. Binge Eating Scale (BES)

3. Gormally, Black, Daston \& Rardin

4. Barratt Impulsiveness Scale-11 (BIS_11)
} 
شاخصهاى مركزى و براكندكى و در بخش آمار استنباطى از

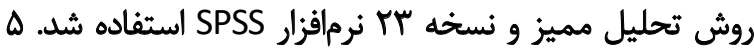

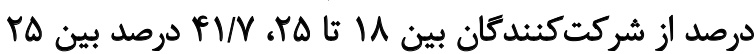

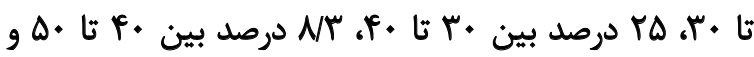

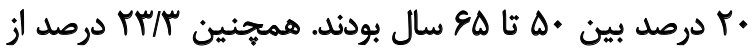

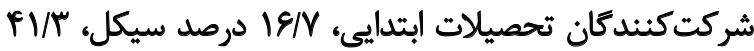

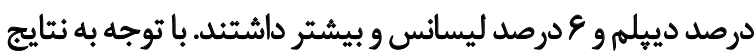

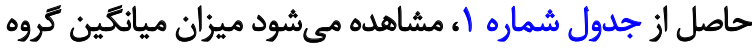

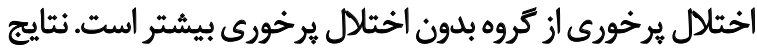
كامل به طور دقيق در جدول شرو بدون اختاره آمده است.

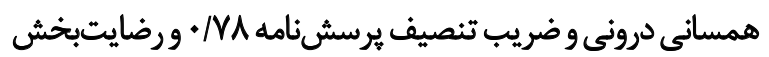

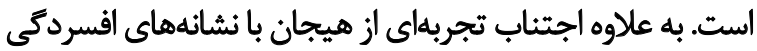

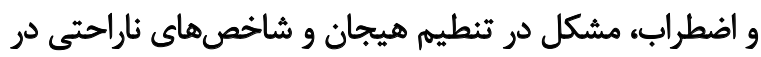

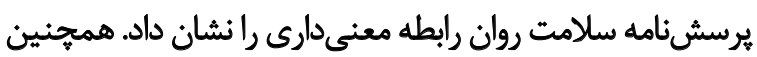

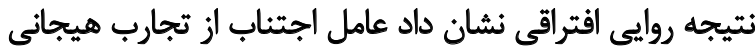

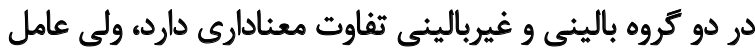

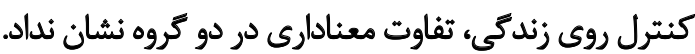

يافتهها

براى تجزيه و تحليل دادهها در بخش آمار توصيفى از جدول ا. ميانكين و انحراف استاندارد خردهآزمونها در دو كروه بدون اختلال برخورى و با اختلال برخورى

\begin{tabular}{|c|c|c|c|}
\hline ميانكين||نحراف استاندارد & متغيرها & \multicolumn{2}{|c|}{ 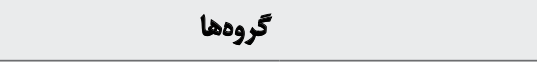 } \\
\hline WE $\pm N r$ & اجتاب هيجانى & \multirow{2}{*}{ اجتناب تجربهاي } & \\
\hline$I r / N \pm r / N$ & كتترل - & & \\
\hline$W T \pm \Psi / N$ & تكانشكر شناختى & \multirow{3}{*}{ ت تكانشكرى } & بلدون اختلال يرخورى \\
\hline$r / / \pm f / \Delta$ & تكانشكر حركى & & \\
\hline$r N a \pm r / \Delta$ & تكانشكر بقيرنامكى & & \\
\hline$r / q \pm N A$ & اجتناب هيجانى & اجتناب تجربهاي & با اختلال بيرخورى \\
\hline$r / \mathscr{T} \pm r / \Lambda$ & كتترل & \multirow{4}{*}{ تكاثشكرى } & \\
\hline Wa土w/G & تكانشكر شناختى & & \\
\hline$T r / T \pm N r$ & تكانشكر حركى & & \\
\hline$r V / E \pm F / r$ & تكانشكر بیىبنامكى & & \\
\hline$r \cdot / \pm N g$ & اجتناب هيجانى & \multirow{2}{*}{ اجتناب تجربهاى } & \multirow{5}{*}{ كل } \\
\hline $19 / \backslash \pm r / \Lambda$ & كتترل & & \\
\hline WE \pm w/ & تكانشكر شناختى & \multirow{3}{*}{ تكانشكرى } & \\
\hline$r r / g \pm g / f$ & تكانشكر حركتى & & \\
\hline$r / \varphi \pm f / g$ & تكانشكر بیيبرنامكى & & \\
\hline
\end{tabular}

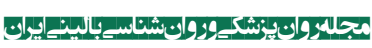

جدول r. تحليل واريانس به تفكيك براى مؤلفهها

\begin{tabular}{|c|c|c|c|c|c|}
\hline سطح معنادارى & dfr & df $)$ & $\mathbf{F}$ & لامبداي ويلكز & متغير \\
\hline $.1 \cdot P^{*}$ & $\Delta A$ & 1 & M/Tr. &.$/ 984$ & اجثناب هيجانى \\
\hline $.1 .1 V^{*}$ & $\Delta A$ & 1 & r/qIr &.$/ Q 8 \wedge$ & كنترل \\
\hline . Arve & $\Delta$ & 1 & $+/ 010$ & $+/ 991$ & تكانشكر شناختى \\
\hline.$/ 419$ & $\Delta A$ & 1 & . PAN &.$/ 997$ & تكائشكر حركتى \\
\hline $1 . .8^{* *}$ & $\Delta$ & 1 & $N \cdot M$ & - /AVA & تكانشك بي برنامكي \\
\hline
\end{tabular}


جدول r. خلاصه تابع مميزى و برخى از اصطلاحات مهم تابع

\begin{tabular}{|c|c|}
\hline تابع & اصطلاحات مهم مربوط به تحليل مميز \\
\hline MIFAA & مقدار ويثره \\
\hline $1 .$. & درصد واريائس \\
\hline $1 .$. & درصد ثراكمي \\
\hline$\cdot / M$ & همبستكى متعارف \\
\hline$+/ \pi 10$ & ل ل امبداى ويلكز \\
\hline W/899 & مجذور كاى \\
\hline 10 & درجه آزادى \\
\hline.$\ldots$ & معنادارى تابع مميز \\
\hline
\end{tabular}

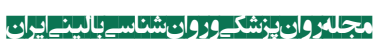

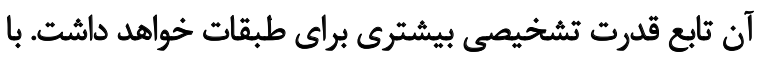

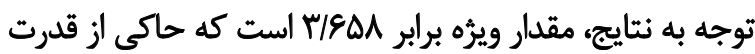

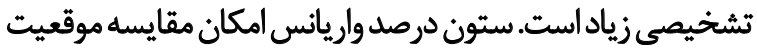

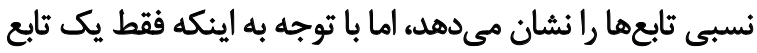

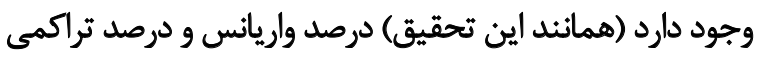

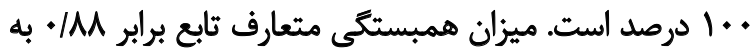

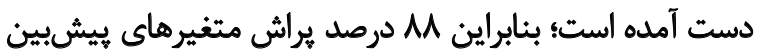

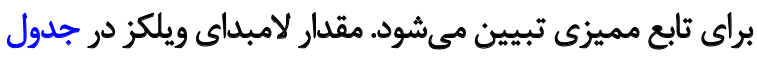

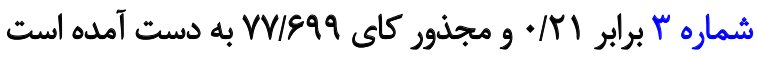

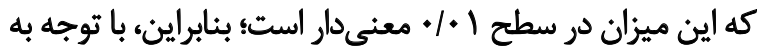

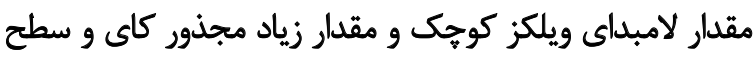

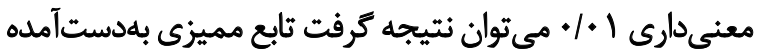

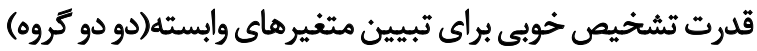

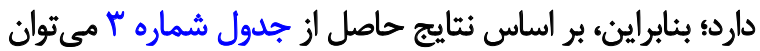

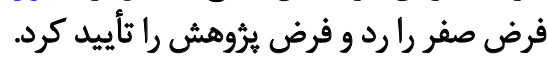

ماتريس ساختار، مقياس متفاوتى از ميزان كمك هركدام از

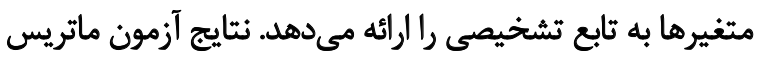

جدول شماره ب نتايج تحليل واريانس به تفكيك براى مؤلفهها

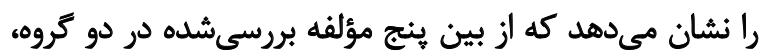

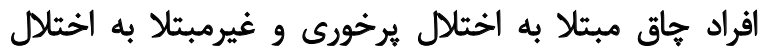

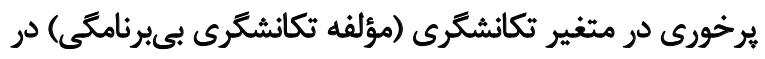

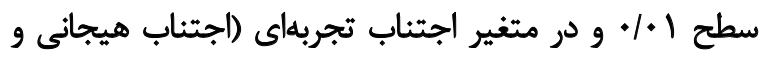

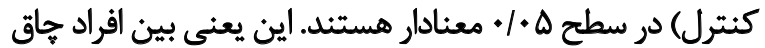

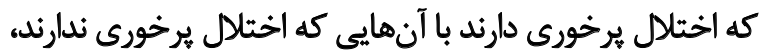

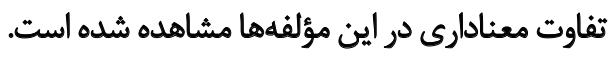

جدول شماره ب خلاصه ثابع مميزى و برخى از اصطلاحات

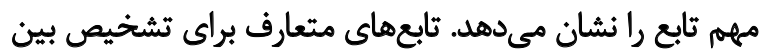

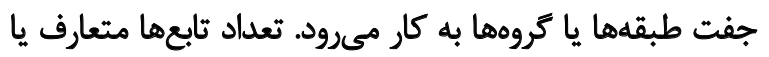

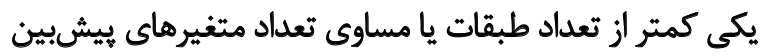

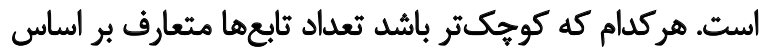

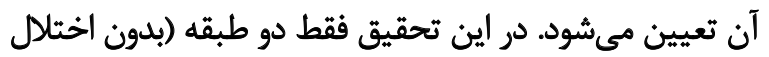

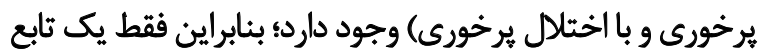
محاسبه شـ. به طور كلى هرجه مقدار ويثه و درصدواريانس ثابع بيشتر باشد، مبه.

جدول F. ماتريس همبستكي تكانشكرى با مؤلفهماى اجتناب تجربهاى

\begin{tabular}{|c|c|c|}
\hline ضرايب & متغيرها & توابع \\
\hline$-+/ 190$ & تكانشكر بي برنامكى & \\
\hline$\cdot N+r$ & اجتناب هيجانى & \\
\hline-+1.90 & كتترل & تابع ا \\
\hline 1.49 & تكانشكر شناختى & \\
\hline $.1 .+1$ & تكانشكر حركتى & \\
\hline
\end{tabular}


جدول ه. نتايج طبقهبثدى

\begin{tabular}{|c|c|c|c|}
\hline \multirow{2}{*}{ 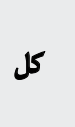 } & \multicolumn{2}{|c|}{ عضويت در كروه ييشبينى شده } & \multirow{2}{*}{ كروه } \\
\hline & با اخثلال يرخورى & بدون اختلال يرخورى & \\
\hline$m$ & 1 & M & بدون اختلال يرخورى \\
\hline r & TV & • & با اختلالل يرخورى \\
\hline $.11 .$. & $\cdot / \mu$ &.$/ 97$ & بدون اختلال يرخورى \\
\hline$. / 1+$ &.$/$. & - & با اختلال يرخورى \\
\hline \multicolumn{3}{|c|}{ 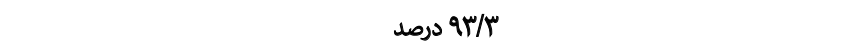 } & ييشبينى عضويت كروهى \\
\hline
\end{tabular}

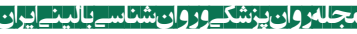

اين اختلال، ناتوانى مبتلايان در كنترل خود و ناتوانيى در توجه بـ به

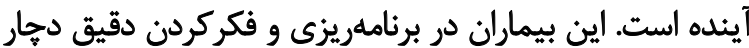

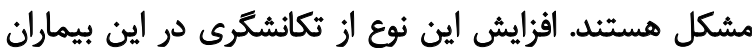

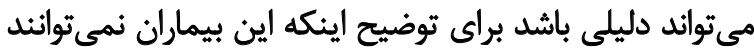

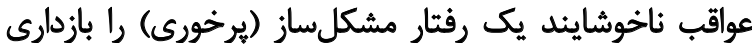

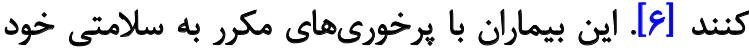

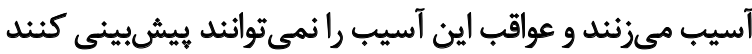

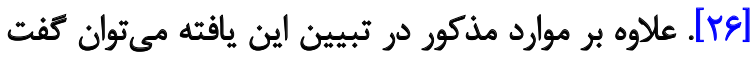

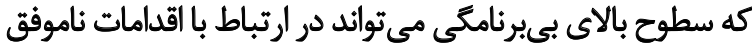

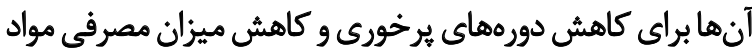

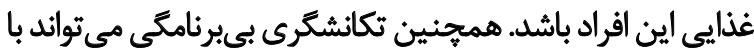
رزيمهاى غذايى ناموفق اين افراد در ارتباط باشد.

درنهايت، در اجتناب تجربهاي نمره هر دوخر درهمقياس اجتناب

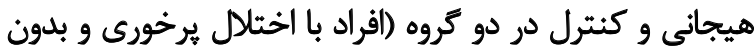

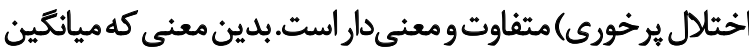

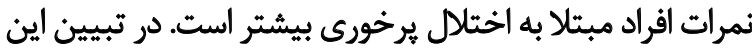

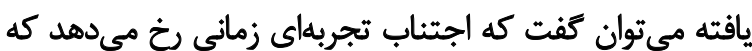

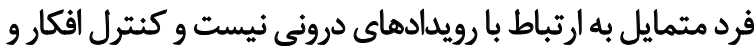

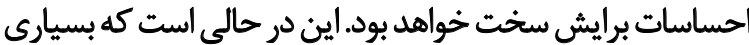

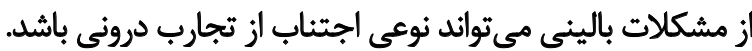

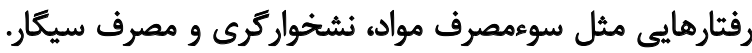

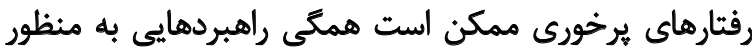

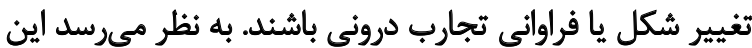

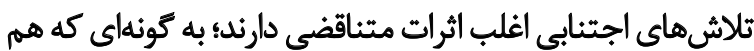

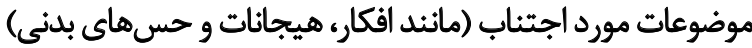
را افزايش مى دهد و هم نار احتى روانشئاختى بيشترى به به همراه

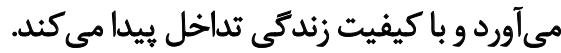

ياسخهاى اجتنابى اغلب از طريق كاهش فورى ناراحتى

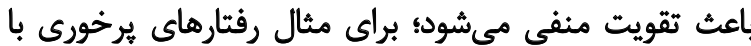

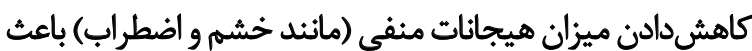

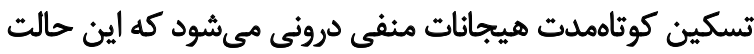

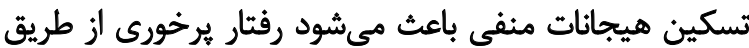

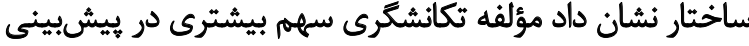

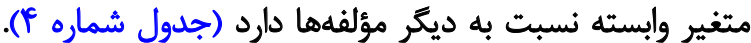

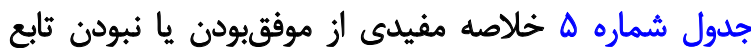

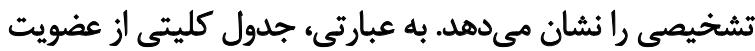

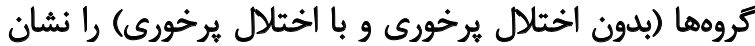

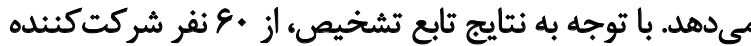

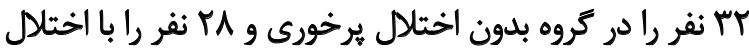

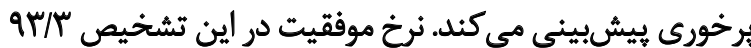

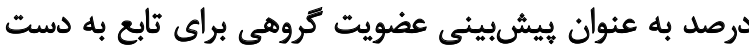

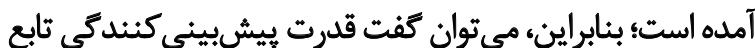

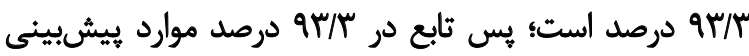
درستى براى عضويت و يا طبقهبندى افراد در كروهها دارد.

ث

نتايج بررسى مؤلفهها به تفكيك حاكى از آن است كه از بين

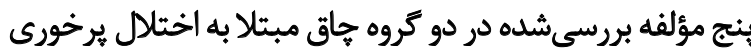

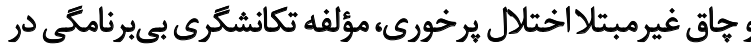

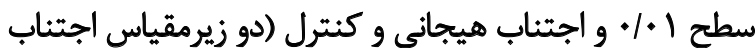

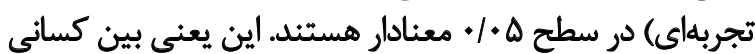

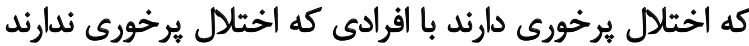

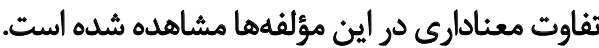

همجنين ماتريس ساختار نشان داد مؤلفه تكانشكرى

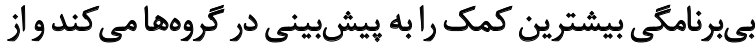

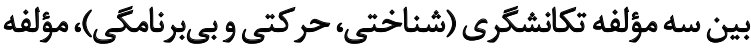

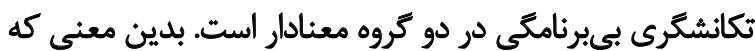

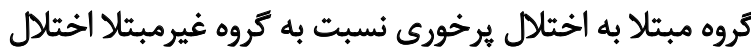

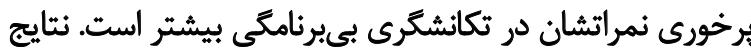

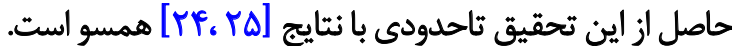

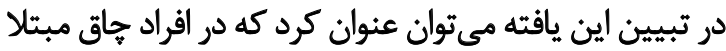

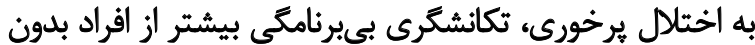

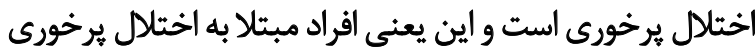

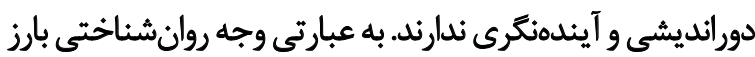




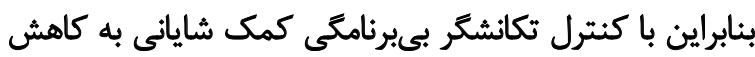

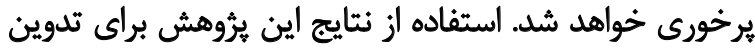

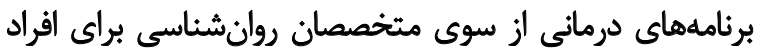

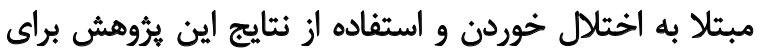

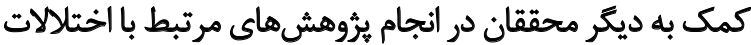
خوردن از ييشنهادات كاربردى اين مطالعه است.

\section{ملاحظات اخلاقى \\ يبيروى ازو الصول الخالاق يُوهش}

همه اصول اخلاقي در اين مقاله رعايت شده است است

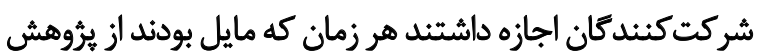

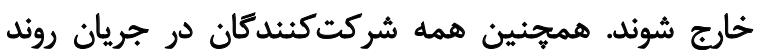

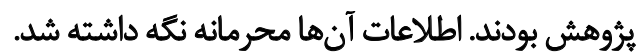

$$
\text { مامي مالي }
$$

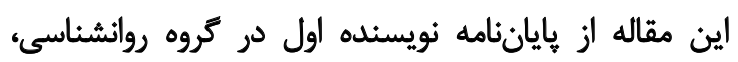

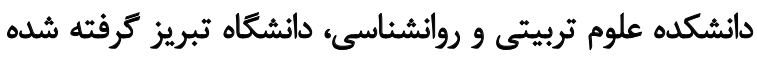

$$
\text { مشار كتنويسند مكان }
$$

تمام نويسندكان در آمادهسازى اين مقاله مشاركت داشتهاند.

$$
\text { ثارض مثأف }
$$

بنابر اظهار نويسندكان، اين مقاله تعارض منافع ندارد.
بازدارى هيجان منفى، تقويت شوده بنابراين، مصرف مواد غذائي

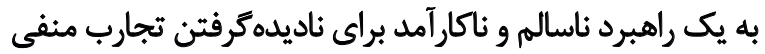

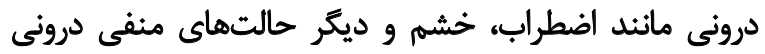

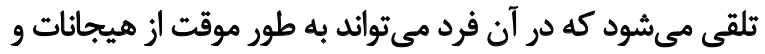

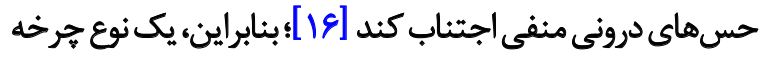

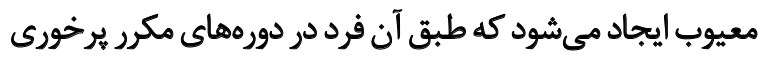

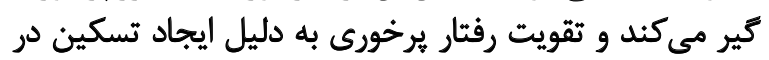

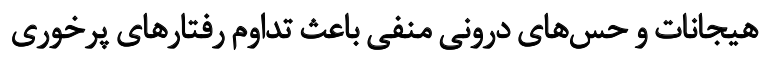

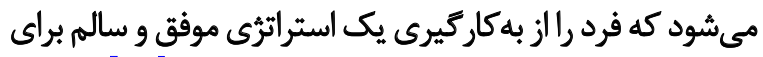

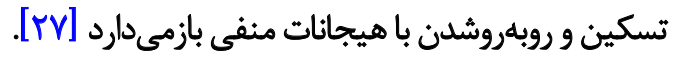

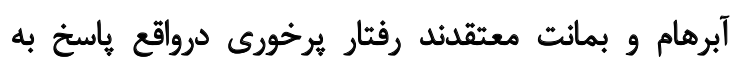

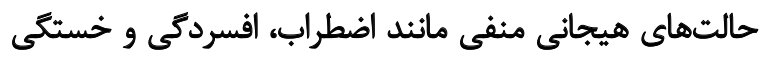

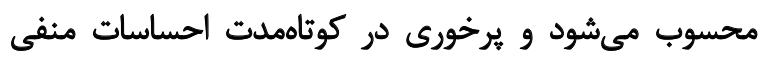

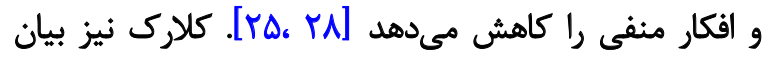

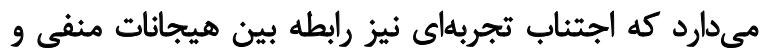

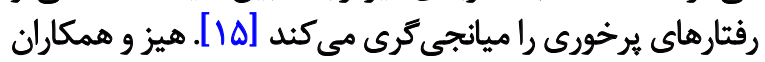

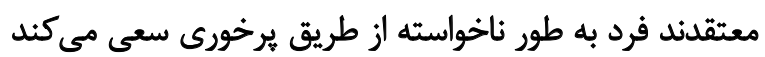

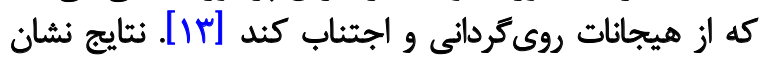

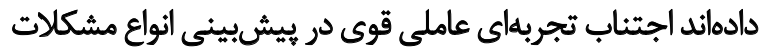

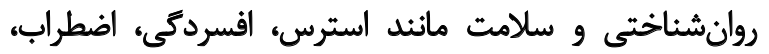
سيعاركشيدن و يرخورى است [| [ f]]

\section{نتيجلهَيرى}

درمجموع مى توان نتيجه كرفت بر اساس متغير هاى تكانشكرى

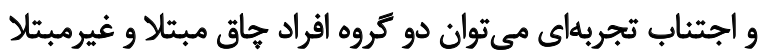

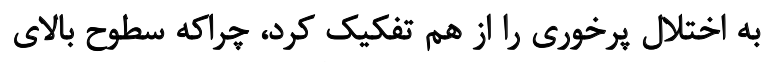

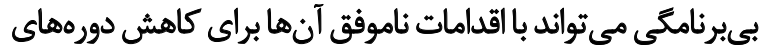

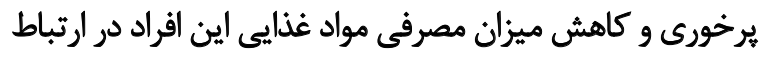

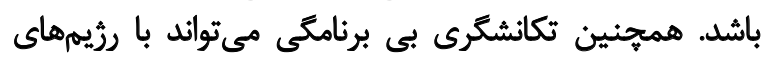

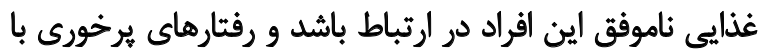

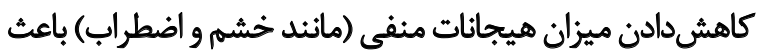

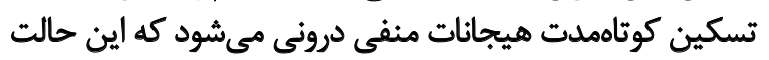

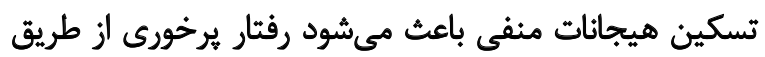
بازدارى هيجان منفى، تقويت شود.

خود نزارشىبودن مقياس ها، كافىنبودن تعداد اعضاى نمونه و

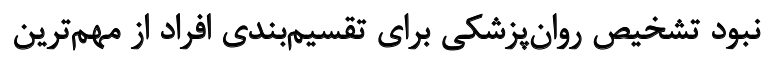

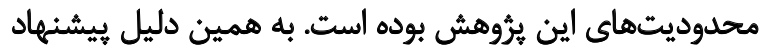

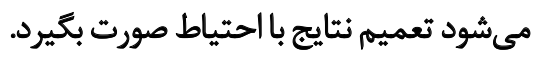

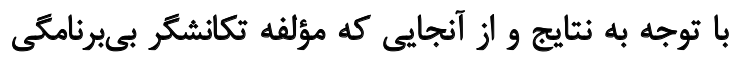

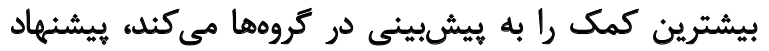

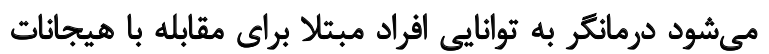

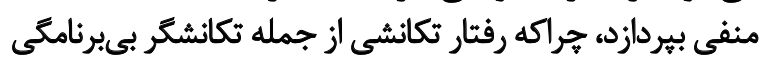

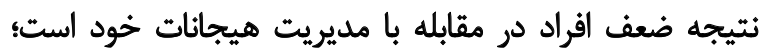




\section{References}

[1] The American Psychiatric Association. Diagnostic and statistical manual of mental disorders (DSM-5®). Philadelphia: The American Psychiatric Association; 2013. [DOI:10.1176/ appi.books.9780890425596]

[2] Bertoli S, Leone A, Ponissi V, Bedogni G, Beggio V, Strepparava MG, et al. Prevalence of and risk factors for binge eating behaviour in 6930 adults starting a weight loss or maintenance programme. Public Health Nutrition. 2016; 19(1):71-7. [DOI:10.1017/S1368980015001068] [PMID]

[3] Kianmanesh S, Jenaabadi H. The study of difference between the frequency of positive and negative symptoms of schizophrenia in people with different cultural backgrounds. Global Journal of Scientific Researches. 2014; 2(5):124-7.

[4] Bulik CM, Sullivan PF, Wade TD, Kendler KS. Twin studies of eating disorders: A review. International Journal of Eating Disorders. 2000; 27(1):1-20. [DOI:10.1002/ (SICI)1098-108X(200001)27:13.0.CO;2-Q]

[5] Leehr EJ, Krohmer K, Schag K, Dresler T, Zipfel S, Giel KE. Emotion regulation model in binge eating disorder and obesity-a systematic review. Neuroscience \& Biobehavioral Reviews. 2015; 49:125-34. [DOI:10.1016/j.neubiorev.2014.12.008] [PMID]

[6] Karimpour Vazifehkhorani A, Bakhshipour Roodsari A, Kamali Ghasemabadi H, Etemadi Chardah N. [Effectiveness of reward-based task on affective levels of depressed individuals (Persian)]. Iranian Journal of Psychiatry and Clinical Psychology. 2019; 24(1):6-15. [DOI:10.29252/nirp. ijpcp.24.1.6]

[7] Mahmood Aliloo M, Hashemi Nosratabad T, Karimpour Vazifehkhorani A. [The role of impulsivity, sensitivity to reward and anhedonia in distinction people with symptoms of borderline personality disorder from ordinary people (Persian)]. Iranian Journal of Psychiatry and Clinical Psychology. 2018; 24(2):136-47. [DOI:10.32598/ijpcp.24.2.136]

[8] Stanford MS, Mathias CW, Dougherty DM, Lake SL, Anderson NE, Patton JH. Fifty years of the barratt impulsiveness scale: An update and review. Personality and Individual Differences. 2009; 47(5):385-95. [DOI:10.1016/j. paid.2009.04.008]

[9] Meule A, Platte P. Attentional bias toward high-calorie food-cues and trait motor impulsivity interactively predict weight gain. Health Psychology Open. 2016; 3(1):2055102916649585. [DOI:10.1177/2055102916649585] [PMID] [PMCID]

[10] Claes L, Vandereycken W, Vertommen H. Impulsivityrelated traits in eating disorder patients. Personality and Individual Differences. 2005; 39(4):739-49. [DOI:10.1016/j. paid.2005.02.022]

[11] Kachooei M, Hasani J, Amrollahi Nia M. [Comparison of impulsivity and difficulties in emotion regulation among overweight women with and without binge eating disorder (Persian)]. Iranian Journal of Endocrinology and Metabolism. 2016; 17(5):391-401.

[12] Hayes SC. Acceptance and commitment therapy, relational frame theory, and the third wave of behavioral and cognitive therapies. Behavior Therapy. 2004; 35(4):639-65. [DOI:10.1016/S0005-7894(04)80013-3]

[13] Hayes SC, Strosahl KD, Wilson KG. Acceptance and commitment therapy. New York City: Guilford Press; 1999

[14] Hayes SC, Wilson KG, Gifford EV, Follette VM, Strosahl K. Experiential avoidance and behavioral disorders: A functional dimensional approach to diagnosis and treatment. Journal of Consulting and Clinical Psychology. 1996 64(6):1152-68. [DOI:10.1037/0022-006X.64.6.1152] [PMID]

[15] Clark MM, Abrams DB, Niaura RS, Eaton CA, Rossi JS. Self-efficacy in weight management. Journal of Consulting and Clinical Psychology. 1991; 59(5):739-44. [DOI:10.1037/0022-006X.59.5.739] [PMID]

[16] Brockmeyer T, Skunde M, Wu M, Bresslein E, Rudofsky G, Herzog W, et al. Difficulties in emotion regulation across the spectrum of eating disorders. Comprehensive Psychiatry. 2014; 55(3):565-71. [DOI:10.1016/j. comppsych.2013.12.001] [PMID]

[17] Gross JJ, Jazaieri H. Emotion, emotion regulation, and psychopathology: An affective science perspective. Clinical Psychological Science. 2014; 2(4):387-401 [DOI:10.1177/2167702614536164]

[18] Gormally J, Black S, Daston S, Rardin D. The assessment of binge eating severity among obese persons. Addictive Behaviors. 1982; 7(1):47-55. [DOI:10.1016/0306-4603(82)900247] [PMID]

[19] Moloodi R, Dezhkam M, Mootabi F, Omidvar N. [Comparison of early maladaptive schema in obese binge eaters and obese non-binge eaters (Persian)]. International Journal of Behavioral Sciences. 2010; 4(2):109-14.

[20] Moeller FG, Barratt ES, Dougherty DM, Schmitz JM Swann AC. Psychiatric aspects of impulsivity. American Journal of Psychiatry. 2001; 158(11):1783-93. [DOI:10.1176/ appi.ajp.158.11.1783] [PMID]

[21] Javid M, Mohammadi N, Rahimi C. [Psychometric properties of an Iranian version of the Barratt Impulsiveness Scale-11 (BIS-11) (Persian)]. Scientific Journal of Psychological Models Methods 2. 2012; 2(8):21-32.

[22] Bond FW, Hayes SC, Baer RA, Carpenter KM, Guenole $\mathrm{N}$, Orcutt HK, et al. Preliminary psychometric properties of the acceptance and action questionnaire-II: A revised measure of psychological inflexibility and experiential avoidance. Behavior Therapy. 2011; 42(4):676-88. [DOI:10.1016/j. beth.2011.03.007] [PMID]

[23] Abasi E, Fti L, Molodi R, Zarabi H. [Psychometric properties of Persian version of acceptance and action questionnaire-II (Persian)]. Journal of Psychological Models and Methods. 2013; 3(10):65-80.

[24] Gearhardt AN, White MA, Masheb RM, Morgan PT, Crosby RD, Grilo CM. An examination of the food addiction construct in obese patients with binge eating disorder. International Journal of Eating Disorders. 2012; 45(5):65763. [DOI:10.1002/eat.20957] [PMID] [PMCID]

[25] Brownell KD, Walsh BT. Eating disorders and obesity: A comprehensive handbook. New York City: Guilford Publications; 2017. 
[26] Hege M, Stingl K, Kullmann S, Schag K, Giel K, Zipfel S, et al. Attentional impulsivity in binge eating disorder modulates response inhibition performance and frontal brain networks. International Journal of Obesity. 2015; 39(2):35360. [DOI:10.1038/ijo.2014.99] [PMID]

[27] Van Strien T, Ouwens MA. Effects of distress, alexithymia and impulsivity on eating. Eating Behaviors. 2007; 8(2):251-7. [DOI:10.1016/j.eatbeh.2006.06.004] [PMID]

[28] Abraham SF, Beumont P. How patients describe bulimia or binge eating. Psychological Medicine. 1982; 12(3):625-35. [DOI:10.1017/S0033291700055732] 